\title{
Comprensión hermenéutica y análisis situacional en Karl R. Popper ${ }^{1}$
}

\author{
José de Lira Bautista \\ Departamento de Filosofía \\ Universidad Autónoma de Aguascalientes \\ jdelira@correo.uaa.mx
}

El desarrollo de la filosofía de la ciencia, desde la segunda mitad del siglo XIX y a lo largo del siglo XX, además de su propio dinamismo, marcado por las tesis centrales del empirismo, se vio afectado por otras corrientes y autores cuyas ideas se planteaban de manera alternativa y contrapuesta a las empiristas, originando así una serie de polémicas sobre la naturaleza, los métodos y el progreso de la ciencia, entre otros temas. La hermenéutica rescata del olvido (generado por el entusiasmo empirista en las leyes generales de la ciencia natural), el conocimiento, manifestado en forma de comprensión, de lo individual, de lo irrepetible, de lo temporal (asociados con el hombre y la sociedad), y los propone como objeto de estudio de las ciencias sociales y las humanidades.

Así, a pesar de las grandes diferencias entre las tradiciones empirista y hermenéutica en la filosofía de la ciencia, sus caminos van a tener puntos de encuentro en su desarrollo. Uno de esos puntos está en el giro hermenéutico de la filosofía de la ciencia popperiana, que enfrentaba el problema de la racionalidad del método de contrastación de las teorías.

En efecto, el método falsacionista de Popper presentaba una serie de problemas metodológicos resaltados por sus críticos, entre ellos Neurath y Kuhn que, entre otras cosas, consistían en señalar, apoyándose en el convencionalismo de Duhem, que las hipótesis,

${ }^{1}$ Esta ponencia fue presentada originalmente en el duodécimo Congreso Mundial de Filosofía realizado en Seúl (Corea) en julio y agosto de 2008. 
en su conjunto, son infalsables. Esta situación lleva a Popper a considerar que la investigación científica no consiste únicamente en la contrastación de teorías, sino en todo el proceso de investigación tomado en su conjunto, que va desde el planteamiento de problemas, pasando por la contrastación empírica, hasta el surgimiento de nuevos problemas. Esta ampliación de la contrastación está sustentada en la lógica situacional, que introduce la hermenéutica en la teoría de la ciencia popperiana.

Popper elabora su aportación a la hermenéutica a partir de la tesis del mundo tres. A diferencia de la mayor parte de los pensadores hermeneutas, traslada el objeto de la comprensión del segundo al tercer mundo: lo que se comprende son los contenidos de pensamiento objetivo. Pero aunque la comprensión sea un proceso psicológico, perteneciente al segundo mundo, su resultado, la interpretación, pertenece al tercer mundo. Por eso, la comprensión sólo se puede entender en su relación con el tercer mundo ${ }^{2}$ y la equipara con el esquema de resolución de problemas por medio del método de conjeturas y refutaciones, cuya forma más simple es la siguiente:

$$
P_{1} \rightarrow T T \rightarrow E E \rightarrow P_{2}
$$

en donde $P_{1}$ es el problema del que se parte; TT es la teoría tentativa o el primer intento de interpretación; $E E$ es la eliminación de errores o análisis crítico de la teoría tentativa; y $P_{2}$ es la nueva situación problemática surgida de la crítica ejercida sobre la situación problemática anterior. Sobre este proceso «se logrará una comprensión satisfactoria, si la interpretación, la teoría conjetural encuentra apoyo en el hecho de poder arrojar nueva luz sobre nuevos problemas; o encuentra apoyo en el hecho de explicar muchos subproblemas, algunos de los cuales no veíamos al comienzo. Así podemos decir que es posible evaluar el progreso realizado comparando $P_{1}$ con algunos problemas posteriores (digamos, $\left.P_{n}\right){ }^{3}$.

2 Cf. Karl POPPER, Conocimiento objetivo, trad. Carlos Solís Santos, Madrid: Tecnos 1982, 155-156 pp.

3 POPPER, Conocimiento objetivo, p. 157. 
Popper identifica el proceso de comprensión con el de resolución de problemas, según el cual los problemas surgen del trasfondo (background) del tercer mundo, de tal manera que al problema, junto con su trasfondo, le llama situación problemática; el trasfondo está constituido por el lenguaje y otros supuestos teóricos ${ }^{4}$ entre los que resalta la tradición de primer orden, es decir, por el conocimiento que tenemos disponible en un momento dado y del que surgen los problemas. La comprensión, de esta manera, se convierte en una comprensión de la situación problemática, lo cual, en contra tal vez del mismo Popper, nos lleva a la cuestión del contexto de descubrimiento, rechazada por él mismo, debido a que no se trata de un proceso lógico.

Popper sostiene que su lógica de la situación es consecuente con la comprensión hermenéutica ${ }^{5}$. El aspecto distintivo de la lógica de la situación frente a la comprensión consiste en remitirla al mundo tres; los humanistas como Dilthey y Collingwood proponen una idea de la comprensión que se ubica en el mundo dos popperiano, es decir, en el mundo de los estados subjetivos de las personas. Al llevar el problema de la comprensión al mundo tres, Popper señala que pareciera que se aleja del dogma fundamental de los humanistas, es decir, del «dogma de que los objetos de nuestra comprensión pertenecen fundamentalmente al segundo mundo o, en todo caso, de que han de ser explicados en términos psicológicos» ${ }^{6}$. Distingue así entre el proceso de la comprensión y el resultado del proceso, que es la interpretación. La interpretación como tal es una teoría y por tanto pertenece al tercer mundo; la comprensión, en cambio, al ser un proceso, implica actividades subjetivas, sin embargo, sólo puede entenderse en su relación con objetos del tercer mundo, de tal manera que aunque el proceso de comprensión sea subjetivo, se refiere, o está engarzado, con objetos del tercer mundo ${ }^{7}$.

${ }^{4}$ Cf. POPPER, Conocimiento objetivo, 157-158 pp.

5 «En las ciencias sociales existe un método puramente objetivo que puede bien denominarse el método objetivamente comprensivo, o lógica de la situación», en: Karl POPPER, «La lógica de las ciencias sociales», en: Theodor W. ADORNO et. al., La disputa del positivismo lógico en la sociología alemana, Barcelona-México: Grijalbo 1973, Vigésimo quinta tesis.

6 POPPER, Conocimiento objetivo, p. 155.

${ }^{7}$ Cf. POPPER, Conocimiento objetivo, p. 155-156 pp. 
Al criticar a Dilthey porque no logra distinguir esos grados de la comprensión, Popper anota que «he de comprender con certeza todo enunciado si es que comprendo el lenguaje» ${ }^{8}$, independientemente de la verdad del enunciado, pues la comprensión encierra una competencia lingüística del que comprende. En cambio, cuando Dilthey distingue grados de comprensión, reservando para el más elevado de ellos la interpretación, deja desprotegidos, por así decir, los de nivel más bajo. Hay que tener en cuenta que la comprensión, para Dilthey, constituye el método propio de las ciencias del espíritu y que el objeto de la comprensión son las manifestaciones de vida ajenas, teniendo como base la vivencia y la comprensión de uno mismo. Por medio de estas ciencias «es posible elevar la comprensión de lo singular a plano de objetividad $\gg$.

Popper ejemplifica sus acuerdos y diferencias con los humanistas con la comparación que realiza entre el análisis situacional y el método de la evocación subjetiva de Collingwood: «Collingwood pone el acento en la situación de un modo muy semejante a como yo hago hincapié en lo que denomino situación problemática» ${ }^{10}$, es decir, el punto en el que Popper y Collingwood están de acuerdo es en el de la situación problemática, pues ambos proponen la reconstrucción de la situación en la que se generan los problemas pero con distinta finalidad, pues, como anota Popper, «Collingwood deja bien claro que lo esencial al comprender la historia no es el análisis de la situación misma, sino el proceso que tiene lugar en la mente del historiador y que consiste en la evocación, en la repetición simpatética de la experiencia original» ${ }^{11}$. Para Popper, como ya hemos expuesto, el análisis de la situación tiene como meta comprender una situación histórica específica por medio de un metaproblema, de una metaconjetura y una metasolución, y no la evocación de experiencias pasadas. «Por tanto, qua historiador, lo que tiene que hacer no es evocar experiencias pasadas, sino dispo-

8 POPPER, Conocimiento objetivo, p. $162 \mathrm{n}$.

9 Wilhelm DILTHEY, «El mundo histórico», en: Obras Completas, Vol. VII, México: FCE 1978, p. 321.

10 DILTHEY, «El mundo histórico», p. 177.

11 DILTHEY, «El mundo histórico», p. 177. 
ner argumentos objetivos en pro y en contra de su análisis conjetural de la situación» ${ }^{12}$.

Si bien la diferencia de la lógica de la situación de Popper respecto de la comprensión hermenéutica de Collingwood y de Dilthey estriba principalmente en la aceptación o rechazo del mundo de los contenidos de pensamiento objetivo, esta diferencia parece que se anularía respecto de la hermenéutica de Gadamer. En efecto, para Gadamer, la comprensión no trata de revivir o evocar la experiencia del interpelado, sino que busca comprender el contenido verdadero de la expresión de su vivencia ${ }^{13}$. Esto es posible en Gadamer desde la perspectiva del lenguaje, pues la hermenéutica se resuelve en el medio del lenguaje en donde adquiere objetividad la comprensión misma. Por eso, «el lenguaje es el medio universal en el que se realiza la comprensión misma. La forma de realización de la comprensión es la interpretación ${ }^{14}$. Lenguaje, tradición y comprensión están así indisolublemente unidas ${ }^{15}$.

Podemos concluir este trabajo señalando la relevancia que tiene la comprensión hermenéutica en la filosofía de la ciencia popperiana, la cual le abre una posibilidad de solucionar el problema de la racionalidad de la ciencia. Tradicionalmente se ve en el modelo de contrastación de Popper un modelo bastante simplificado. Sin embargo, al considerarlo en su conjunto representa un esfuerzo enorme por darle un sustento racional a la metodología de la investigación. La lógica situacional, equiparada con el método de comprensión hermenéutica, le permite explicar no solamente la contrastación, sino la generación de nuevas teorías, aun en contra de sus cuestionamientos a los procesos de descubrimiento científico.

12 DILTHEY, «El mundo histórico», p. 177.

${ }^{13}$ Cf. Hans-Georg GADAMER, Verdad y método, Vol. I, Salamanca: Sígueme 1997, p. 461.

14 GADAMER, Verdad y método, p. 467.

15 Cf. GADAMER, Verdad y método, p. 485. 


\section{RESUMEN}

En este trabajo se expone el giro hermenéutico en la filosofía de la ciencia popperiana, el cual representa un hito en la búsqueda de la racionalidad científica, pues permite explicar y comprender de manera más adecuada el método de contrastación de las teorías así como el desarrollo de las mismas. De manera particular, la incorporación de parámetros propiamente hermenéuticos, elaborados desde la perspectiva de Popper, como lo es la lógica situacional, sustentada por la teoría del mundo tres y la concepción sobre la tradición científica, abre una puerta a la comprensión de la racionalidad de la ciencia, ampliando el campo de la concepción ingenua de la falsación de teorías, al desarrollo mismo de las teorías, que a su vez va desde el planteamiento de problemas, las propuestas de solución y la eliminación de errores, a la generación de nuevos problemas de investigación. Uno de los resultados nos conduce a replantear nuestra concepción de la filosofía de la ciencia de Popper desde una perspectiva cercana a la hermenéutica filosófica.

Palabras clave: Popper; falsación; contrastación; giro hermenéutico; filosofía de la ciencia.

\section{ABSTRACT}

In this paper the author exposes the hermeneutic turn in Popperian philosophy of science. It is a milestone in the search of scientific rationality, because permit us explain and understand both the method of deductive test of theories and the growth of knowledge. Especially, incorporating hermeneutics parameters, build up from Popper's point of view, like situational logic, supported on the third world theory and the scientific tradition theory, open a door to another form of understand the scientific rationality. It expands the ingenuous model of falsation towards the question of increase of knowledge, which consists on pose problems, put forwards tentative theories, evaluate error elimination, and generate new problems. One of the outcomes leads us to replant our conception of Popper's philosophy of science close from a hermeneutic point of view.

Key words: Popper; falsation; test of theories; hermeneutic turn; philosophy of science. 